\title{
Immediate Manifestation of Ocular Decompression Retinopathy following Anterior Chamber Paracentesis
}

\author{
Jamie Prince David Fleischman \\ Department of Ophthalmology, University of North Carolina School of Medicine, \\ Chapel Hill, NC, USA
}

\section{Keywords}

Ocular decompression retinopathy · Paracentesis · Primary open-angle glaucoma

\begin{abstract}
Ocular decompression retinopathy (ODR) is a complication of rapid lowering of intraocular pressure (IOP) resulting in hemorrhages in multiple retinal layers. We report a case of ODR that developed within minutes following anterior chamber paracentesis in an adult female with primary open-angle glaucoma. A 61-year-old Black woman with primary open-angle glaucoma presented with marked elevation of IOP (46 mm Hg in the right eye and $30 \mathrm{~mm} \mathrm{Hg}$ in the left) despite maximal medical therapy and bilateral selective laser trabeculoplasty. Vision in the right eye decreased from counting fingers at 3 feet 1 week earlier to no light perception at the time of presentation. Anterior chamber paracentesis was performed on the right eye to reduce IOP and stabilize the eye until further surgical intervention could be performed. IOP in the right eye decreased to $6 \mathrm{~mm} \mathrm{Hg}$ postoperatively. Within minutes of the procedure, several intraretinal blot hemorrhages appeared in the periphery of the right eye. During this time, visual acuity remained at no light perception. The findings were consistent with decompression retinopathy. By 12 weeks postoperatively, the ODR had resolved with visual acuity of light perception and normal fundoscopy except for profound cupping. Our case demonstrates how rapidly ocular decompression can form following IOP reduction.

(C) 2019 The Author(s)

Published by S. Karger AG, Basel
\end{abstract}




\section{Case Reports in Ophthalmology}

\section{Introduction}

Ocular decompression retinopathy (ODR), first described in 1992, is an uncommon complication of intraocular pressure (IOP)-lowering interventions [1]. Decompression retinopathy presents with hemorrhages occurring in all retinal layers. ODR has been reported following both medical and surgical procedures and in various types of glaucoma including congenital glaucoma [2]. Previously reported cases of ODR have occurred up to 14 days postoperatively [3]. The majority of cases are asymptomatic or present with mildly diminished visual acuity and self-resolve within a few weeks.

Anterior chamber paracentesis is a method to lower IOP acutely, usually used under complicated circumstances. Patients undergoing ocular paracentesis are at increased risk of developing ODR due to the rapid decrease in IOP [4].

We report the case of a 61-year-old woman who developed ODR immediately after an uncomplicated anterior chamber paracentesis.

\section{Case Report}

A 61-year-old Black female with no significant past medical history was referred to our clinic for further management of severe primary open-angle glaucoma in both eyes. Prior to presentation at our clinic, the patient was placed on maximum medical therapy (500 $\mathrm{mg}$ of acetazolamide orally twice a day, brimonidine $0.2 \%$-timolol $0.5 \%$ twice a day and latanoprost $0.005 \%$ at bedtime). She had undergone selective laser trabeculoplasty 6 years earlier. She presented to her local eye care provider for complaints of decreased vision without pain. During this visit, she was noted to have IOP readings of $45 \mathrm{~mm} \mathrm{Hg}$ in the right eye and $35 \mathrm{~mm} \mathrm{Hg}$ in the left. Her best corrected vision was found to be counting fingers at 3 feet in the right eye and $20 / 25$ in the left. She was urgently referred to our clinic but was unable to follow up until 3 weeks later due to transportation issues.

On examination, her best corrected visual acuity in her right eye had decreased to no light perception, and her left eye remained at 20/25. Both eyes showed significantly elevated IOP - $46 \mathrm{~mm} \mathrm{Hg}$ in the right eye and $30 \mathrm{~mm} \mathrm{Hg}$ in the left. A dilated ophthalmoscopic exam was normal except for cupping of the nerves. The cup-to-disc ratio was found to be 0.99 in the right eye and 0.95 in the left. Given the recent visual loss in the right eye, we hoped to restore vision by performing an anterior chamber paracentesis to lower her baseline IOP while also administering maximal aqueous suppression therapy. This would allow the pharmacological reduction of aqueous humor formation and maintenance of a lower IOP until further surgical intervention could be performed in the coming week.

The patient was currently taking acetazolamide $250 \mathrm{mg}$ PO BID from the referring provider. The anterior chamber paracentesis was performed and IOP immediately dropped to $6 \mathrm{~mm} \mathrm{Hg}$ in the right eye. A drop of brimonidine and dorzolamide-timolol was then applied $5 \mathrm{~min}$ apart. No more than 5 min postoperatively, several new intraretinal blot hemorrhages appeared in the periphery of the right eye (Fig. 1). No preretinal or subretinal hemorrhages were noted. No hemorrhages were appreciated in the left eye. IOP increased to $15 \mathrm{~mm} \mathrm{Hg}$ $30 \mathrm{~min}$ after the procedure. Visual acuity in the right eye remained at no light perception.

One week later, the patient underwent implantation of an Ahmed glaucoma valve in the right eye and ab interno trabeculotomy with a trabectome in the left eye. On postoperative day 1 ( 7 days after paracentesis), fundoscopy revealed unchanged intraretinal hemorrhages in the inferior mid-periphery of the right eye (Fig. 2). Visual acuity improved to light 


\section{Case Reports in Ophthalmology}

perception in the right eye and remained at 20/25 in the left. Eight weeks postoperatively, she developed a hypertensive phase and underwent cyclophotocoagulation. She developed choroidal effusions following this intervention. Twelve weeks postoperatively, peripheral ophthalmoscopic evaluation was repeated and the patient's choroidal detachments had resolved. Additionally, the retina was found to be normal without hemorrhage. More than 2 years following this presentation, the patient has undergone multiple surgeries but her vision has improved to finger counting with eccentric fixation.

\section{Discussion}

ODR is a rare, iatrogenic complication of lowering IOP [1]. ODR is characterized by a transient hemorrhagic retinopathy, with the majority of cases remaining asymptomatic or presenting with mildly diminished visual acuity. The intraretinal hemorrhages typically selfresolve within a few weeks. Although initial cases were described after trabeculectomy, ODR has been reported after both medical and surgical IOP-lowering interventions. Only a few cases have been identified after glaucoma surgery, causing only $3.0 \%$ of reported cases [4]. Here, we describe a patient with severe-stage primary open-angle glaucoma who rapidly developed ODR after treatment with anterior chamber paracentesis.

To our knowledge, our case represents the most rapid onset of decompression retinopathy in a glaucoma patient. A recent systematic review reported 15\% of cases of ODR developing on the operative day, with all cases having had a mean time to diagnosis of $1.5 \pm 2.0$ days [3]. Of the reported cases, only one decompression retinopathy occurred within minutes of paracentesis [5]. This intervention was performed for macular branch artery occlusion.

Proposed mechanisms for ODR include mechanical and vascular changes. Regular IOP preserves the shape of the globe and the normal corneal-scleral curvature. Therefore, postintervention hypotony may lead to distortion of the sclera, and the loss of this support structure may result in many downstream physiologic changes, including increased capillary fragility [5]. Alternatively, loss of autoregulation has been proposed as a cause of ODR [5]. Autoregulation is a mechanism by which organs self-regulate blood flow in the context of changing perfusion pressure. Ocular conditions can affect local vascular constriction or dilation in response to changes in IOP. Interventions which acutely lower IOP may decrease arterial resistance and induce increased retinal blood flow. This transient increase can cause blood flow leakage through fragile capillaries. Long-standing elevated IOP, such as in our patient with primary open-angle glaucoma, may cause dysfunction of retinal autoregulation and lead to development of decompression retinopathy [6].

Risk factors for the development of ODR after IOP-lowering treatment include elevated preoperative IOP, a substantial decrease in IOP from preoperative baseline, and hematologic factors such as an increased prothrombin time and activated partial thromboplastin time and decreased hemoglobin/hematocrit [3]. Our patient was not under the care of a primary care physician and did not have any lab work on file. She was, however, taking $81 \mathrm{mg}$ aspirin daily, which should not have any effect on clotting factors. Thus, our patient's risk factors were an elevated preoperative IOP ( $46 \mathrm{~mm} \mathrm{Hg}$ ) and a substantial postoperative decrease in IOP $(6 \mathrm{~mm}$ $\mathrm{Hg}$ ). The patient presented for preoperative assessment and was found to be in good health.

Our case demonstrates the rapid onset of ODR that can occur after anterior chamber paracentesis. Prior to undergoing an IOP-lowering procedure, it is important to assess risk factors for ODR. Decompression retinopathy is a rare, self-limiting condition that can develop during the postoperative period. 


\section{Statement of Ethics}

The authors have no ethical conflicts to disclose. The subject gave informed consent.

\section{Disclosure Statement}

The authors have no conflicts of interest to declare.

\section{Funding Sources}

The authors did not receive funding.

\section{Author Contributions}

D. Fleischman examined the patient and edited the paper. J. Prince wrote and edited the paper.

\section{References}

1 Fechtner RD, Minckler D, Weinreb RN, Frangei G, Jampol LM. Complications of glaucoma surgery. Ocular decompression retinopathy. Arch Ophthalmol. 1992 Jul;110(7):965-8.

2 Ramtohul P, Chardavoine M, Beylerian M, Aziz A, Matonti F, Denis D. Decompression retinopathy following nonpenetrating deep sclerectomy for primary congenital glaucoma. BMC Ophthalmol. 2018 Sep;18(1):240.

3 Mukkamala SK, Patel A, Dorairaj S, McGlynn R, Sidoti PA, Weinreb RN, et al. Ocular decompression retinopathy: a review. Surv Ophthalmol. 2013 Nov-Dec;58(6):505-12.

4 Jung KI, Lim SA, Lopilly Park HY, Park CK. Risk factors for decompression retinopathy after glaucoma surgery. J Glaucoma. 2014 Dec;23(9):638-43.

5 Gupta R, Browning AC, Amoaku WM. Multiple retinal haemorrhages (decompression retinopathy) following paracentesis for macular branch artery occlusion. Eye (Lond). 2005 May;19(5):592-3.

6 Moore D, Harris A, Wudunn D, Kheradiya N, Siesky B. Dysfunctional regulation of ocular blood flow: A risk factor for glaucoma? Clin Ophthalmol. 2008 Dec;2(4):849-61. 


\section{Case Reports in Ophthalmology}

www.karger.com/cop

Prince and Fleischman: ODR following Anterior Chamber Paracentesis

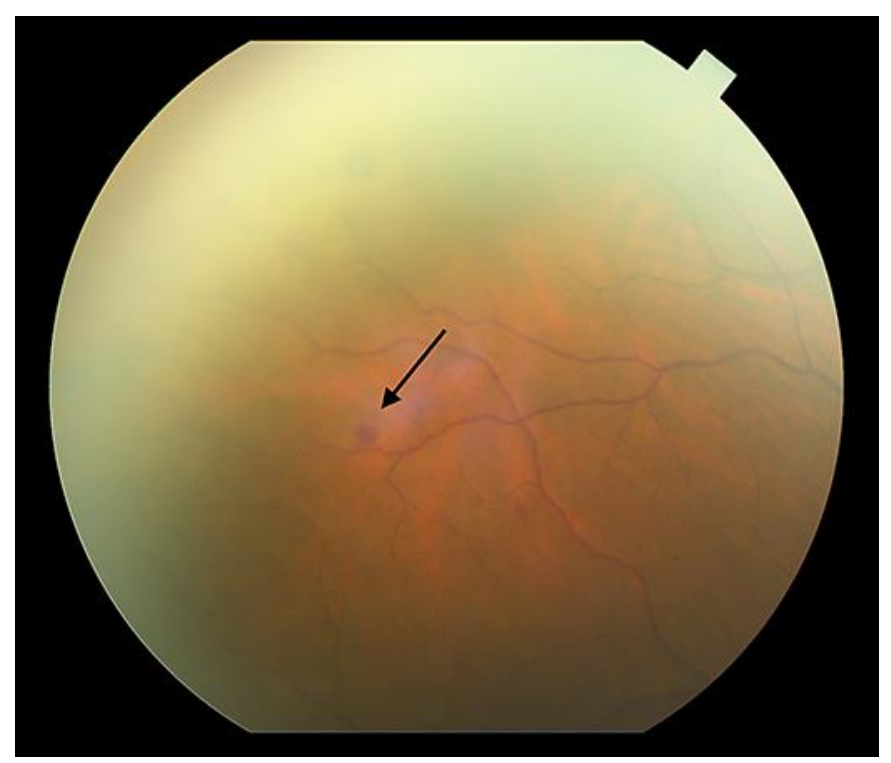

Fig. 1. Color fundus photograph of the far temporal periphery of the right eye. The arrow points to one of a cluster of intraretinal hemorrhages appreciated immediately following a paracentesis. There were no hemorrhages prior to the paracentesis. The view is limited by a multifactorial keratopathy.

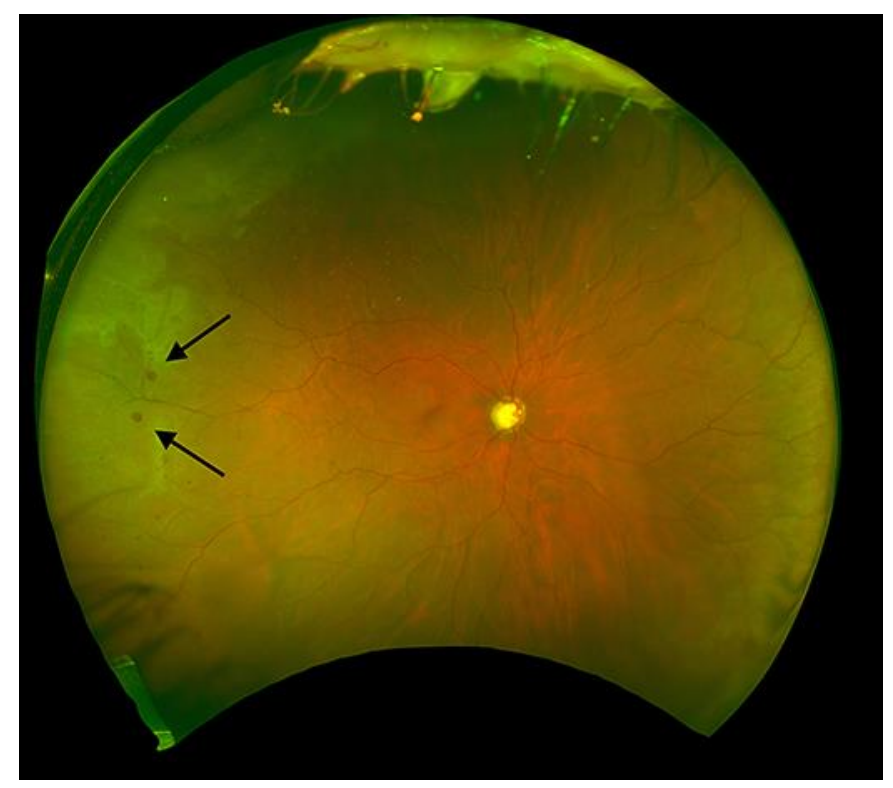

Fig. 2. Optos ${ }^{\circledR}$ wide-field fundus photograph of the right eye 1 week after paracentesis. A cluster of intraretinal hemorrhages can be appreciated in the far temporal periphery (arrows). These appeared unchanged to the hemorrhages noted immediately following the paracentesis. 